\title{
Improving the Management of Atrial Fibrillation in General Practice: Protocol for a Mixed Methods Study
}

Simon de Lusignan ${ }^{1,2}$, MSc, MD, FRCP; F D Richard Hobbs ${ }^{1}$, FRCP; Harshana Liyanage ${ }^{1}$, PhD; Filipa Ferreira ${ }^{1}$, $\mathrm{PhD}$; Manasa Tripathy ${ }^{1}$, MSc; Neil Munro ${ }^{1}$, DPhil; Michael Feher ${ }^{1}$, MD, FRCP; Mark Joy ${ }^{1}$, PhD

${ }^{1}$ Nuffield Department of Primary Care Health Sciences, University of Oxford, Oxford, United Kingdom

${ }^{2}$ Royal College of General Practitioners Research and Surveillance Centre, London, United Kingdom

Corresponding Author:

Simon de Lusignan, MSc, MD, FRCP

Nuffield Department of Primary Care Health Sciences

University of Oxford

Eagle House, Walton Well Road

Oxford, OX2 6ED

United Kingdom

Phone: 441865289344

Email: simon.delusignan@phc.ox.ac.uk

\section{Abstract}

Background: Atrial fibrillation (AF) is one of the commonest arrhythmias observed in general practice. The thromboembolic complications of AF include transient ischemic attack, stroke, and pulmonary embolism. Early recognition of AF can lead to early intervention with managing the risks of these complications.

Objective: The primary aim of this study is to investigate if patients are managed in general practice according to current national guidelines. In addition, the study will evaluate the impact of direct oral anticoagulant use with respect to AF complications in a real-world dataset. The secondary aims of the study are to develop a dashboard that will allow monitoring the management of AF in general practice and evaluate the usability of the dashboard.

Methods: The study was conducted in 2 phases. The initial phase was a quantitative analysis of routinely collected primary care data from the Oxford Royal College of General Practitioners Research and Surveillance Center (RCGP RSC) sentinel network database. AF cases from 2009 to 2019 were identified. The study investigated the impact of the use of anticoagulants on complications of AF over this time period. We used this dataset to examine how AF was managed in primary care during the last decade. The second phase involved development of an online dashboard for monitoring management of AF in general practice. We conducted a usability evaluation for the dashboard to identify usability issues and performed enhancements to improve usability.

Results: We received funding for both phases in January 2019 and received approval from the RCGP RSC research committee in March 2019. We completed data extraction for phase 1 in May 2019 and completed analysis in December 2019. We completed building the AF dashboard in May 2019. We started recruiting participants for phase 1 in May 2019 and concluded data collection in July 2019. We completed data analysis for phase 2 in October 2019. The results are expected to be published in the second half of 2020. As of October 2020, the publications reporting the results are under review.

Conclusions: Results of this study will provide an insight into the current trends in management of AF using real-world data from the Oxford RCGP RSC database. We anticipate that the outcomes of this study will be used to guide the development and implementation of an audit-based intervention tool to assist practitioners in identifying and managing AF in primary care.

International Registered Report Identifier (IRRID)： RR1-10.2196/21259

(JMIR Res Protoc 2020;9(11):e21259) doi: $\underline{10.2196 / 21259}$

\section{KEYWORDS}

atrial fibrillation; medical record systems, computerized; general practice; cross-sectional studies; qualitative research 


\section{Introduction}

Atrial fibrillation (AF) is one of the commonest arrhythmias observed in clinical practice [1]. Globally, the incidence of AF is rising. The thromboembolic complications of AF can lead to significant disabling consequences for patients, including transient ischemic attack, stroke, and pulmonary embolism. Early recognition of AF in general practice can lead to early intervention with managing the risks of these complications.

Current guidelines on the management of AF by the National Institute for Health and Care Excellence (NICE) advise identifying and managing the underlying causes of $\mathrm{AF}$, treating the arrhythmia, and assessing and managing the risk of stroke in these patients [2]. Risk of stroke is determined by the $\mathrm{CHA}_{2} \mathrm{DS}_{2}$-VASc (Congestive heart failure, Hypertension, Age, Diabetes, previous Stroke/transient ischemic attack-VAScular disease) assessment tool [3], which guides the practitioner on commencing anticoagulation treatment to manage this risk. The risk of starting a patient on anticoagulation is assessed using the HAS-BLED (Hypertension, Abnormal renal/liver function, Stroke, Bleeding history or predisposition, Labile international normalized ratio, Elderly, Drugs/alcohol concomitantly) score, assessing for major bleeding risks [4].

Anticoagulation therapy aims to reduce the risk of thromboembolic events. This has been achieved using warfarin (a vitamin $\mathrm{K}$ antagonist) for many years. The introduction of direct oral anticoagulants (DOACs) such as apixaban and rivaroxaban to clinical practice has changed how $\mathrm{AF}$ is managed in clinical practice [5]. In comparison to warfarin, DOACs are observed to have similar or better mortality and vascular outcomes [6]. They have the added benefit of requiring no monitoring, as opposed to warfarin use [7]. Though studies have highlighted that increased number of patients in clinical practice are receiving treatment for AF [8], whether they are managed according to national or local guidelines is unclear. Better understanding of current management trends of AF in population will guide practitioners on how best to employ interventions in clinical practice to increase awareness of $\mathrm{AF}$ and early recognition.

The aim of this study is to understand the disease and prescribing trends related to AF in general practice. We also aim to develop a system to monitor practice-level indicators for AF of the same trends and evaluate the usability of this system. The primary objective of this study is to investigate if people with newly diagnosed AF treated with DOACs versus warfarin have any difference in stroke or all-cause mortality in long-term follow-up. The secondary objectives of this study are to develop an AF dashboard as an intervention method at a practice level and to evaluate the usability of the AF dashboard.

\section{Methods}

\section{Study Design}

This study combines qualitative and quantitative methods and therefore, was planned as a mixed method study [9-11]. It was run within the Oxford Royal College of General Practitioners Research and Surveillance Centre (RCGP RSC) sentinel network of general practices. The mixed method study was conducted in 2 phases. Phase 1 was a quantitative analysis of AF data. We conducted a repeated cross-sectional study to identify those patients diagnosed with AF during 2009-2019 and identified current therapeutic management of their AF. Phase 2 involved development and evaluation of a dashboard for monitoring data quality for AF. The dashboard provides the general practices in the RCGP RSC sentinel network an access to quality indicators related to AF management that are updated weekly.

\section{Phase 1: Quantitative Analysis of AF Data}

\section{Data Source}

The initial phase involved a repeated cross-sectional retrospective analysis of data extracted from the RCGP RSC database, a surveillance network, which collects data from computerized medical records (CMR) from more than 3.5 million patients and over 300 general practices in England. CMRs are used during consultations to record patient conditions and prescriptions [12,13]. Anonymized clinical coded data from participating general practices were uploaded weekly to a secure network at the Clinical Informatics Research Group [12,14]. This study used NICE guidelines as the standard to compare the management of AF across this database.

\section{Case Ascertainment}

We used Read codes and CTV3 codes used in primary care CMR systems to identify patients with AF. Pay-for-performance (P4P) for chronic disease management has improved data quality related to $\mathrm{AF}$ in the recent past [15]. We excluded patients who had a previous stroke and those not on British National Formulary-recommended dose of DOAC. We included patients over 18 years with code for the diagnosis of AF who had been registered at any point between 2008 and 2019.

\section{Exposures and Outcomes}

We considered the exposure as continuous anticoagulant prescription for the first time after receiving an AF diagnosis. Although almost all anticoagulant prescriptions in the United Kingdom are issued from primary care if an anticoagulant is started in a hospital, we measured exposure from the first general practice prescription. We excluded patients who had an interval of greater than 90 days between anticoagulant prescriptions.

Outcomes are first records of stroke and all-cause mortality based on previously published Read codes $[16,17]$ Stroke was included regardless of etiology, an approach used in other studies $[18,19]$. Study participants were followed for stroke and all-cause mortality till the end of the study period.

\section{Covariates}

We included variables likely to be used as indicators in prescribing anticoagulants: age-band, gender, and deprivation reporting Index of Multiple Deprivation (IMD) quintile. IMD is a national measure of socioeconomic status, which can be derived at an individual level from first part of postcode. We adjusted for comorbidities from that part of the stroke risk score $\left(\mathrm{CHA}_{2} \mathrm{DS}_{2}\right.$-VASc), namely, heart failure, hypertension, stroke or transient ischemic attack, and myocardial infarction or 
peripheral vascular disease at baseline. We also included smoking status as a covariate.

\section{Statistical Analysis}

Analysis of data extracted from general practice CMR was carried out using the statistical software R. We studied the influence of the anticoagulation regimen by evaluating the cause-specific hazard ratio and the subdistribution hazard ratios of both events. Additionally, we estimated cumulative incidence for both events by direct regression, utilizing the inverse of the probability of censoring weights method [15] with time-varying effects [20]. We will report and interpret both cause-specific and subdistribution analyses. The cause-specific hazard ratio is often interpreted as estimating etiological association, estimating associations between covariates, and the rate at which events occur in those participants who are event-free. The cause - specific hazard ratio can be interpreted as a rate ratio. Whenever the proportional hazards assumption was violated, we interpreted all hazard ratios as time-averaged effects.

We will determine the (adjusted) incidence and prevalence of AF for each year along with the proportion of incident and prevalent cases treated with nothing, antiplatelet medications only (eg, aspirin), and anticoagulant therapy (warfarin/types of DOACs). We will also determine the number of strokes, transient ischemic attacks, deaths, and bleeds in the incident and prevalent cases per year.

\section{Phase 2: Development of a Dashboard for Monitoring AF}

We developed an online dashboard for the general practices to understand the quality of their data across a variety of AF-related indicators. The AF dashboard provided 4 categories of information: (1) case ascertainment-incidence, prevalence, standardized prevalence, and any indicators related to $\mathrm{P} 4 \mathrm{P}$ prevalence; (2) indications for therapy and risk factors; (3) management choices; and (4) quality. The dashboard was developed using Tableau data visualization software (Salesforce Inc). The initial dashboard is hosted on the public dashboard cloud server and accesses a publicly accessible database server (located within information technology infrastructure of the University of Oxford), which hosts only aggregated data to comply with the requirements of information governance. The dashboard allowed users to view the AF indicators only from their own practice.

We used the Think Aloud method to validate the usability of the AF dashboard. During usability experiment, study participants were instructed to verbalize their thoughts while concurrently conducting predefined tasks on the dashboard [21]. The design of the Think Aloud experiment involved participants engaging in 5 tasks. Participants were asked to verbalize their thought process while engaging in each of the tasks [22]. During the initial 4 tasks, the participants interacted with the 4 main sections of the dashboard. They observed the given information and interpreted it with respect to that particular aspect of $\mathrm{AF}$ management in their practice. As a fifth task, participants considered the overall dashboard and described the overall state of AF management in their practice in comparison to the RCGP RSC sentinel network.

\section{Participants}

We invited staff from general practices to cover a range of roles from primary care, including general practitioners, nurses, and practice managers. We provided remote participation for those who could not attend in person. We aimed to invite staff from practices participating in the RCGP RSC sentinel network. Our target sample size was about 30 participants based on the guidelines from previous studies [23].

\section{Data Capture and Analysis}

We recorded participant feedback and screen activity using GoToMeeting screen sharing software, version 10.5 (LogMeIn Inc). The audio component of the recordings was exported and transcribed by a professional transcription service. The transcripts were analyzed using NVivo, version 12 (QSR International), a qualitative analysis software. We followed a 3-step approach to analyze the transcripts.

1. Mapping verbalized tasks to sections: Each verbalized task description was extracted and mapped to the corresponding section of the dashboard. A "verbalized task" is the narrative description provided by a participant when interacting with particular component in the dashboard. We organized the verbalized tasks according to the template given in (Table $\mathrm{S} 1$ in Multimedia Appendix 1). The verbalized tasks were mapped to the dashboard sections, and similar feedbacks were grouped.

2. Mapping verbalized tasks to usability problem classes: For each section, we mapped the verbalized tasks to matching usability problem classes. We adapted the usability problem classification method used by Peute et al [21] and identified occurrences for each usability problem class (Table S2 in Multimedia Appendix 1). We also classified the identified verbalized tasks based on whether they were positive feedback, negative feedback, or suggestions for new features.

3. Summarizing usability issues across sections/usability problem class: The completed templates were analyzed to determine the critical sections and key usability problem classes that required attention.

\section{Ethical Considerations}

For phase 1, approval was granted by the research committee of the RCGP RSC. The study did not meet the requirements for a formal ethics board review as per the NHS Health Research Authority's research decision tool [24]. The study was conducted in line with the REporting of studies Conducted using Observational Routinely-collected Data (RECORD) guidelines [25]. For phase 2, personal data were not collected from the study participants. Participants were informed that their verbal responses and screen activities would be recorded. All participants received oral and written information about the study.

\section{Dissemination}

The protocol and data produced from this work will be submitted for publication in a peer-reviewed journal covering the domains of cardiology, epidemiology, and primary care. Additional opportunities include presentation at seminars, conferences, and meetings. 


\section{Results}

We received funding for both phases in January 2019 and received approval from RCGP RSC research committee in March 2019. We completed data extraction for phase 1 in May 2019 and completed analysis in December 2019. We completed building the AF dashboard in May 2019. We started recruiting participants for phase 1 in May 2019 and concluded data collection in July 2019. We completed data analysis for phase 2 to in October 2019. As of October 2020, the publications reporting the results are under review.

\section{Discussion}

The RCGP RSC sentinel network has a large dataset, which is representative of information regarding primary care practices on management of AF. The quantitative analysis will report on the current trends of anticoagulant prescribing using a real-world data set in the United Kingdom and the overall population effect of the use of DOAC use.
Missing/incomplete data could be a potential issue in this analysis. We intend to reduce the effect of this issue by incorporating an ontological approach, considering practice level variations in the anticoagulation prescribing.

The qualitative analysis of the dashboard will indicate the priority of sections that require an improved user experience. We intend to enhance the dashboard using the outcomes of the usability study and deploy to general practices in the RCGP RSC sentinel network.

As the incidence of AF has increased over the years, there is a greater focus on early recognition of AF in general practice to allow for early intervention. This study will give insights into the current trends in management of AF using real-world data from the Oxford RCGP RSC database. We anticipate that the outcomes of this study will be used to guide the development and implementation of an audit-based intervention tool for use in primary care settings to assist practitioners in identifying and managing AF.

\section{Acknowledgments}

The work was supported by Daiichi Sankyo. The funder did not have any involvement in study design, access to data, or influence in this analysis.

\section{Conflicts of Interest}

FDRH has received occasional fees from Bayer and Boehringer Ingelheim for speaking or consulting on atrial fibrillation-related stroke risk.

\section{Multimedia Appendix 1}

Supplementary file with Table 1 and Table 2 described in the study design of phase 2. [DOCX File, 15 KB-Multimedia Appendix 1]

\section{References}

1. Ganz L, Spragg D. Epidemiology of and risk factors for atrial fibrillation. UpToDate. 2015. URL: https://www.uptodate.com/ contents/epidemiology-of-and-risk-factors-for-atrial-fibrillation [accessed 2020-10-17]

2. Friberg L, Rosenqvist M, Lip GYH. Evaluation of risk stratification schemes for ischaemic stroke and bleeding in 182678 patients with atrial fibrillation: the Swedish Atrial Fibrillation cohort study. Eur Heart J 2012 Jun;33(12):1500-1510. [doi: 10.1093/eurheartj/ehr488] [Medline: 22246443]

3. Chugh SS, Havmoeller R, Narayanan K, Singh D, Rienstra M, Benjamin EJ, et al. Worldwide epidemiology of atrial fibrillation: a Global Burden of Disease 2010 Study. Circulation 2014 Feb 25;129(8):837-847 [FREE Full text] [doi: 10.1161/CIRCULATIONAHA.113.005119] [Medline: 24345399]

4. Zhu W, He W, Guo L, Wang X, Hong K. The HAS-BLED Score for Predicting Major Bleeding Risk in Anticoagulated Patients With Atrial Fibrillation: A Systematic Review and Meta-analysis. Clin Cardiol 2015 Sep;38(9):555-561 [FREE Full text] [doi: 10.1002/clc.22435] [Medline: 26418409]

5. Hernandez I, Zhang Y, Saba S. Comparison of the Effectiveness and Safety of Apixaban, Dabigatran, Rivaroxaban, and Warfarin in Newly Diagnosed Atrial Fibrillation. Am J Cardiol 2017 Nov 15;120(10):1813-1819. [doi: 10.1016/j.amjcard.2017.07.092] [Medline: 28864318]

6. Ruff CT, Giugliano RP, Braunwald E, Hoffman EB, Deenadayalu N, Ezekowitz MD, et al. Comparison of the efficacy and safety of new oral anticoagulants with warfarin in patients with atrial fibrillation: a meta-analysis of randomised trials. Lancet 2014 Mar 15;383(9921):955-962. [doi: 10.1016/S0140-6736(13)62343-0] [Medline: 24315724]

7. Lip GYH. Atrial fibrillation in 2011: Stroke prevention in AF. Nat Rev Cardiol 2011 Dec 20;9(2):71-73. [doi: 10.1038/nrcardio.2011.203] [Medline: 22182957]

8. Adderley NJ, Ryan R, Nirantharakumar K, Marshall T. Prevalence and treatment of atrial fibrillation in UK general practice from 2000 to 2016. Heart 2019 Jan;105(1):27-33. [doi: 10.1136/heartjnl-2018-312977] [Medline: 29991504]

9. Teddlie C, Tashakkori A. Overview of Contemporary Issues in Mixed Methods Research. In: Tashakkori A, Teddlie C, editors. Thousand Oaks, CA: Sage Publications; 2010:1-42. 
10. Creswell JW, Clark VLP. Designing and Conducting Mixed Methods Research. Thousands Oak, CA: Sage Publications; 2017.

11. Saunders M, Lewis P, Thornhill A. Research Methods for Business Students. Essex: Pearson Education; 1996.

12. de Lusignan S, van Weel C. The use of routinely collected computer data for research in primary care: opportunities and challenges. Fam Pract 2006 Apr;23(2):253-263. [doi: 10.1093/fampra/cmi106] [Medline: 16368704]

13. Kumarapeli P, de Lusignan S. Using the computer in the clinical consultation; setting the stage, reviewing, recording, and taking actions: multi-channel video study. J Am Med Inform Assoc 2013 Jun;20(e1):e67-e75 [FREE Full text] [doi: 10.1136/amiajnl-2012-001081] [Medline: 23242763]

14. de Lusignan S, Metsemakers JF, Houwink P, Gunnarsdottir V, van der Lei J. Routinely collected general practice data: goldmines for research? A report of the European Federation for Medical Informatics Primary Care Informatics Working Group (EFMI PCIWG) from MIE2006, Maastricht, The Netherlands. Inform Prim Care 2006;14(3):203-209 [FREE Full text] [doi: 10.14236/jhi.v14i3.632] [Medline: 17288707]

15. Kiran T, Hutchings A, Dhalla IA, Furlong C, Jacobson B. The association between quality of primary care, deprivation and cardiovascular outcomes: a cross-sectional study using data from the UK Quality and Outcomes Framework. J Epidemiol Community Health 2010 Oct;64(10):927-934. [doi: 10.1136/jech.2009.098806] [Medline: 20820055]

16. Gallagher AM, van Staa TP, Murray-Thomas T, Schoof N, Clemens A, Ackermann D, et al. Population-based cohort study of warfarin-treated patients with atrial fibrillation: incidence of cardiovascular and bleeding outcomes. BMJ Open 2014 Jan 27;4(1):e003839. [doi: 10.1136/bmjopen-2013-003839] [Medline: 24468720]

17. Moran GM, Calvert M, Feltham MG, Ryan R, Marshall T. A retrospective cohort study to investigate fatigue, psychological or cognitive impairment after TIA: protocol paper. BMJ Open 2015 May 03;5(4):e008149. [doi: 10.1136/bmjopen-2015-008149] [Medline: 25941191]

18. Allan V, Banerjee A, Shah AD, Patel R, Denaxas S, Casas J, et al. Net clinical benefit of warfarin in individuals with atrial fibrillation across stroke risk and across primary and secondary care. Heart 2017 Feb;103(3):210-218. [doi:

10.1136/heartjnl-2016-309910] [Medline: 27580623]

19. Go AS, Mozaffarian D, Roger VL, Benjamin EJ, Berry JD, Borden WB, American Heart Association Statistics CommitteeStroke Statistics Subcommittee. Heart disease and stroke statistics--2013 update: a report from the American Heart Association. Circulation 2013 Jan 01;127(1):e6-e245 [FREE Full text] [doi: 10.1161/CIR.0b013e31828124ad] [Medline: 23239837]

20. Ambrogi F, Scheike TH. Penalized estimation for competing risks regression with applications to high-dimensional covariates. Biostatistics 2016 Oct;17(4):708-721. [doi: 10.1093/biostatistics/kxw017] [Medline: 27118123]

21. Peute LWP, de Keizer NF, Jaspers MWM. The value of Retrospective and Concurrent Think Aloud in formative usability testing of a physician data query tool. J Biomed Inform 2015 Jun;55:1-10 [FREE Full text] [doi: 10.1016/j.jbi.2015.02.006] [Medline: 25733166]

22. Ericsson K, Simon H. Protocol analysis: Verbal reports as data. Cambridge, Massachusetts: MIT Press; 1984.

23. Boddy CR. Sample size for qualitative research. Qualitative Mrkt Res: An Int J 2016 Sep 12;19(4):426-432. [doi: 10.1108/qmr-06-2016-0053]

24. Health Research Authority. Is My Study Research. 2020. URL: http://hra-decisiontools.org.uk/ [accessed 2020-10-15]

25. Nicholls SG, Quach P, von Elm E, Guttmann A, Moher D, Petersen I, et al. The REporting of Studies Conducted Using Observational Routinely-Collected Health Data (RECORD) Statement: Methods for Arriving at Consensus and Developing Reporting Guidelines. PLoS One 2015;10(5):e0125620. [doi: 10.1371/journal.pone.0125620] [Medline: 25965407]

\section{Abbreviations}

AF: atrial fibrillation

CHA2DS2-VASc: Congestive heart failure, Hypertension, Age, Diabetes, previous Stroke/transient ischemic attack-VAScular disease

CMR: computerized medical records

DOAC: direct oral anticoagulant

HAS-BLED: Hypertension, Abnormal renal/liver function, Stroke, Bleeding history or predisposition, Labile international normalized ratio, Elderly, Drugs/alcohol concomitantly

IMD: Index of Multiple Deprivation

NICE: National Institute for Health and Care Excellence

P4P: pay-for-performance

RCGP RSC: Royal College of General Practitioners Research and Surveillance Center

RECORD: REporting of studies Conducted using Observational Routinely-collected Data 
Edited by G Eysenbach; submitted 10.06.20; peer-reviewed by F Palmieri, N Pahlavani; comments to author 19.06.20; revised version received 18.08.20; accepted 25.08.20; published 09.11.20

Please cite as:

de Lusignan S, Hobbs FDR, Liyanage H, Ferreira F, Tripathy M, Munro N, Feher M, Joy M

Improving the Management of Atrial Fibrillation in General Practice: Protocol for a Mixed Methods Study

JMIR Res Protoc 2020;9(11):e21259

URL: https://www.researchprotocols.org/2020/11/e21259

doi: $10.2196 / 21259$

PMID: 33164903

(CSimon de Lusignan, F D Richard Hobbs, Harshana Liyanage, Filipa Ferreira, Manasa Tripathy, Neil Munro, Michael Feher, Mark Joy. Originally published in JMIR Research Protocols (http://www.researchprotocols.org), 09.11.2020. This is an open-access article distributed under the terms of the Creative Commons Attribution License (https://creativecommons.org/licenses/by/4.0/), which permits unrestricted use, distribution, and reproduction in any medium, provided the original work, first published in JMIR Research Protocols, is properly cited. The complete bibliographic information, a link to the original publication on http://www.researchprotocols.org, as well as this copyright and license information must be included. 